\title{
Efeito de valores discrepantes em grupos de contemporâneos na predição dos valores genéticos ${ }^{1}$
}

\author{
Mirella Leme Franco Geraldini Sirol ${ }^{2}$, Janaína Conte Hadlich ${ }^{2}$, Liliane Suguisawa ${ }^{2}$, André \\ Rodrigues Abrahão ${ }^{2}$, Henrique Nunes de Oliveira ${ }^{3}$
}

\author{
1 Projeto financiado pela FAPESP. \\ ${ }^{2}$ Doutorando em Nutrição e Produção Animal pela Faculdade de Medicina Veterinária e Zootecnia - Unesp - Botucatu/SP. \\ ${ }^{3}$ Departamento de Melhoramento e Nutrição Animal - FMVZ - Unesp/Botucatu/SP. Caixa Postal: 560. CEP:18618-000.
}

RESUMO - Foram simuladas nove populações, cada uma com cinco replicações da variável ganho médio diário (GMD1) com distribuição normal e média 100, variando o tamanho dos grupos e os desvios-padrão. Cada replicação foi dividida de modo a formar grupos que representariam grupos de contemporâneos (GC) e de progênie dentro de GC. Cada GC tinha dez pais. Obtiveram-se três conjuntos: o conjunto 1 com 1.000 grupos de contemporâneos (GC), cada um com 100 observações e dez observações por pai; o conjunto 2, com 2.500 GC, 40 observações e quatro observações por pai; e o conjunto 3, com 5.000 GC, 20 observações e dois filhos por pai. Em cada população, gerou-se GMD1, a qual foi transformada em outra variável, da seguinte forma: DIAS1 = 100/GMD1. Calcularam-se para cada pai, dentro de cada GC, as contribuições de cada GC ao valor de cada pai, para GMD1 $\left(\mathrm{C}_{\mathrm{x}}\right)$ e DIAS1 $\left(\mathrm{C}_{\mathrm{y}}\right)$. Os efeitos do máximo e da média de DIAS1 no grupo sobre o valor absoluto de Cy foram significativos, mas o $\mathrm{R}^{2}$ foi baixo (máximo de 16\%). O mínimo de DIAS1 não influenciou o valor de $\mathrm{C}_{\mathrm{y}}$. O máximo e o mínimo de GMD1 sobre $\mathrm{C}_{\mathrm{x}}$ foram significativos, mas os $\mathrm{R}^{2}$ foram muito baixos (máximo de 2\%). A média não influenciou $\mathrm{C}_{\mathrm{x}}$. Em grupos de contemporâneos com um animal com valor de GMD muito baixo, o valor de DIAS desse animal será relativamente muito mais alto, o que afetará a média do grupo e os valores de todos os animais do grupo. Esse efeito se refletirá na avaliação de seus pais e será mais uma importante fonte de erros na avaliação genética do rebanho. Assim, a utilização de DIAS em substituição ao GMD como critério de seleção para o melhoramento de bovinos é contra-indicada, pois deverá reduzir a possibilidade de ganho genético para crescimento.

Palavras-chave: critérios de seleção, ganho de peso médio diário, heterogeneidade de variâncias

\section{Effect of outliers in contemporary groups on breeding value prediction}

ABSTRACT - Nine populations, with five replications each, were simulated for average daily gain (GMD1) assuming normal distribution, average 100, and different group sizes as well as standard deviations. Each replication was divided into three different sets of contemporary (CG) and progeny groups. The first set (I) was formed by 1,000 contemporary groups, 100 observations each and 10 progenies/sire. The second set (II) included 2,500 CG, 40 observations and 4 progenies/sire. The third set (III) consisted of 5,000 CG, 20 observations and two progenies/sire. The number of records per group and sire and the number of sires per group varied within the same set. In each population, GMD1 was transformed by DIAS1=100/GMD1. The contribution of CG to sire breeding value predictions were calculated for GMD1 ( $\mathrm{C}_{\mathrm{x}}$ ) and DIAS1 ( $\mathrm{C}_{\mathrm{y}}$ ). The maximum and the average of DIAS1 effects on $\mathrm{C}_{\mathrm{y}}$ absolute value were significant, but the $\mathrm{R}^{2}$ were low (maximum of $16 \%$ ). The minimum value of DIAS1 has not influenced $\mathrm{C}_{\mathrm{y}}$. The maximum and the minimum of GMD1 on $\mathrm{C}_{\mathrm{x}}$ were significant, but the $\mathrm{R}^{2}$ were very low (maximum 2\%). The GMD1 average has not affected $\mathrm{C}_{\mathrm{x}}$. The effect of the minimum value of DIAS1 was important to determine the CG effect on breeding value of sires with progeny in the set. A very low value of GMD1 for an animal corresponds to a high value of DIAS1 and affects the values of the other animals and the CG average. This effect has an impact on the evaluation of their sires and will be an important source of error in the genetic evaluation of the herd. Thus, the replacement of GMD1 by DIAS1 as selection criteria for growth is not recommended because it will reduce the possibility of genetic gain.

Key Words: average daily weight gain, heterogeneity of variance, selection criteria

\section{Introdução}

Segundo Fries et al. (1996), há indícios de que a utilização de dias para atingir determinado peso, como critério de seleção, favorece touros que produzem progênies mais uniformes. Por meio de exemplos numéricos, Ortiz Peña et al. (2000), Carvalheiro et al. (2001) e Muniz et al. (2005) sugeriram que as diferenças entre os critérios de seleção 
ganho de peso médio diário (GMD) e dias para atingir determinado peso (DIAS) poderiam ser atribuídas às diferenças entre a média aritmética e a média harmônica.

Muniz et al. (2001) sugeriram que a utilização de DIAS como critério de seleção reduziria a variabilidade genética da população, o que não interessaria aos selecionadores, mas produziria resultados positivos na produção comercial. A média harmônica teria diversas aplicações, especialmente quando as variáveis apresentam assimetria à direita, isto é, quando incluem observações com valores muito altos. Nas distribuições simétricas, entretanto, e naquelas em que ocorre alguma assimetria à esquerda, seria difícil a média harmônica apresentar vantagens sobre a média aritmética. As observações à direita da média teriam pouca influência sobre a média harmônica, porém, a influência de qualquer observação muito à esquerda praticamente determinaria o valor da média harmônica. Em algumas situações, isto seria muito útil como, por exemplo, para calcular o tamanho efetivo de uma progênie. Nesses casos, em vez de utilizar o somatório do número de filhos de um animal em cada grupo de contemporâneos, utilizar-se-ia o somatório da média harmônica do número de filhos do touro e dos filhos de outros touros em cada grupo de contemporâneos. Assim, a importância daqueles grupos formados por filhos do mesmo touro seria reduzida para avaliação daquele touro.

No entanto, a aplicação da média harmônica não pode ser feita para qualquer situação. É correto afirmar que as médias de DIAS são maiores nos grupos com maior variabilidade. Todavia, no processo de avaliação genética, por qualquer método, não se trabalha diretamente com as médias de todas as progênies dos touros. As soluções das equações de modelos mistos para determinado touro $i$, sem dados próprios de produção e com $n_{i j}$ filhos em cada j-ésimo grupo de contemporâneos com $n_{-i j}$ filhos de outros touros, em relação a uma variável y qualquer, são proporcionais a (Van Vleck \& Boldman, 1993):

$$
\sum_{j-1}^{J}\left(\frac{n_{i j} * n_{-i j}}{n_{i j}+n_{-i j}}\right)\left(\bar{y}_{i j .}-\bar{y}_{-i j .}\right),
$$

em que $J$ = número de grupos de contemporâneos; $\bar{y}_{i j}=$ média dos valores observados dos filhos do touro $i$; $\bar{y}_{-i j .}=$ média dos valores observados dos filhos de outros touros.

Desse modo, o valor genético do touro $i$ é proporcional à média dos filhos dos touros dentro de cada grupo, mas depende da média dos filhos de outros touros no mesmo grupo. O valor genético de um touro é proporcional à somatória dos desvios das médias do touro dentro de cada grupo de contemporâneos. Sabe-se que a média harmônica é influenciada pelo menor valor dentro de cada conjunto e, assim, o desvio de um touro em DIAS em um grupo de contemporâneo é a diferença entre as médias harmônicas de GMD dos filhos do touro e dos filhos de outros touros e, portanto, muito dependente do menor valor de GMD entre os filhos do touro no grupo, mas também do menor valor dos filhos de outros animais no grupo, ou seja, muito sensível à presença de observações discrepantes negativas. Portanto, mesmo que determinado touro tenha valor relativamente mediano para GMD, o fato de estar em um grupo de contemporâneos com um indivíduo discrepante para baixo (em GMD) pode determinar que seu desvio em relação à média do grupo seja extremamente elevado.

Objetivou-se verificar, por simulação, se observações com valores muito baixos em um grupo de contemporâneos poderiam ter influência marcante na avaliação dos touros com filhos naquele grupo.

\section{Material e Métodos}

Foram simulados nove tipos de população com cinco replicações, cada uma com 100.000 observações, todas com distribuição normal. Todas as populações foram geradas com média 100 e os nove tipos de populações variaram quanto ao tamanho dos grupos e aos desvios-padrão. Foram utilizados desvios-padrão de 10, 20 e 30 unidades e as observações foram divididas, aleatoriamente, em grupos de duas categorias que representariam os grupos de contemporâneos e os pais.

No conjunto 1, foram formados 1.000 grupos de contemporâneos (GC), cada um com aproximadamente 100 observações, dez de cada pai. No conjunto 2 , as observações foram distribuídas em 2.500 grupos, cada um com aproximadamente 40 observações e quatro observações para cada pai. O conjunto 3 continha 5.000 grupos de contemporâneos, cada um com 20 observações e dois filhos de cada pai em cada observação.

Como o processo de atribuição das observações aos grupos ou aos pais foi aleatório, o número de observações por grupo e por pai, e mesmo o número de pais por grupo, variou dentro do mesmo conjunto. Cada grupo tinha aproximadamente dez pais. Foram, então, geradas cinco replicações de cada conjunto, com cada um dos três desvios-padrão. Todas foram truncadas de modo que os valores gerados abaixo de dez unidades (10\% da média) foram repostos por outros gerados novamente. Em cada população, a variável gerada (GMD1) foi transformada 
em outra variável (DIAS1) pormeio da função: DIAS1=100/ GMD1.

Foram, então, calculados para cada grupo de contemporâneos a média e o desvio-padrão máximo e mínimo das duas variáveis (GMD1 e DIAS1). Também para GMD1 e DIAS1, foram calculadas, para cada pai, dentro de cada GC, as seguintes estatísticas:

$$
C_{x}=\left(\frac{n_{i j} * n_{-i j}}{n_{i j}+n_{-i j}}\right)\left(\bar{x}_{i j .}-\bar{x}_{-i j . j}\right),
$$

em que $n_{i j}=$ número de filhos do pai $i$ no GC $j ; n_{-i j}=$ número de filhos de outros pais no GC $j ; \bar{x}_{i j}$. = média dos valores observados dos filhos do pai $i$ no GC $j ; \bar{x}_{-i j}=$ média dos valores observados dos filhos de outros pais no GC $j$; $C_{X}=$ a contribuição de cada GC ao valor de cada pai para a variável GMD1; $C_{y}=$ calculada de modo semelhante, no entanto, $C_{y}$ é a contribuição de cada GC ao valor de cada pai para a variável DIAS1.

Se um GC tem valores de $\mathrm{C}_{\mathrm{x}}$ muito altos ou de $\mathrm{C}_{\mathrm{y}}$ muito baixos, influencia muito no valor genético dos animais. Assim, a média dos valores absolutos das contribuições de cada grupo permite estimar a contribuição relativa daquele grupo para os touros com filhos no grupo.

Os valores destas estatísticas foram regredidos nas médias, nos máximos e mínimos das variáveis estudadas no grupo de contemporâneos. Uma vez que em cada grupo o mínimo de GMD1 corresponde sempre ao máximo de DIAS1, optou-se por regredir o valor das estatísticas no mínimo e no máximo da própria variável utilizada.

Adotou-se o sistema de análises estatísticas SAS $^{\circledR}$ (1990) para a realização de todas as análises.

Tabela 1 - Médias do número total de contribuições (Pais x GC), do número de observações por pai em cada grupo (NPPai) e do número de observações por grupo de contemporâneos (NOGC) no conjunto e desviospadrão simulados (DPSIM)

Table 1 - Averages of total number of contributions (Sire $x$ CG), number of observations by sire within group (NPPai), and number of observations by contemporary group (NOGC) for each simulation of standard deviation (DPSIM) and set

\begin{tabular}{lcrrr}
\hline DPSIM & $\begin{array}{c}\text { Conjunto } \\
\text { Set }\end{array}$ & NPPai & NOGC & $\begin{array}{r}\text { Pais } x \text { GC } \\
\text { Sire } x \text { CG }\end{array}$ \\
\hline 30 & 1 & 10,00 & 100,00 & $9.999,6$ \\
20 & 1 & 10,00 & 100,00 & $9.999,8$ \\
10 & 1 & 10,00 & 100,00 & $9.999,8$ \\
30 & 2 & 4,08 & 40,08 & $24.538,8$ \\
20 & 2 & 4,07 & 40,08 & $24.549,6$ \\
10 & 2 & 4,08 & 40,08 & $24.536,6$ \\
30 & 3 & 2,31 & 20,31 & $43.207,2$ \\
20 & 3 & 2,31 & 20,31 & $43.271,4$ \\
10 & 3 & 2,31 & 20,31 & $43.214,4$ \\
\hline
\end{tabular}

\section{Resultados e Discussão}

As médias do número de filhos por touro em cada grupo de contemporâneos e do número de observações por GC para cada conjunto gerado são apresentadas na Tabela 1 . A aleatorização do processo de geração dos grupos de contemporâneos teve como conseqüência uma distribuição bastante irregular dos dados, tal como observado nas populações naturais.

Nas Tabelas 2 e 3 consta o resumo das análises de regressão para $C_{y}$ e $C_{x}$, respectivamente. Os efeitos de máximo e da média de DIAS1 no valor absoluto de $C_{y}$ foram significativos em todas as replicações de todos os conjuntos utilizados. Apesar disso, o $\mathrm{R}^{2}$ da regressão foi baixo na maioria das situações estudadas, evidenciando que muito pouco da variação observada pode ser explicado por este parâmetro. No conjunto 3, em que os dados estavam distribuídos em muitos grupos de contemporâneos e no qual o desvio-padrão simulado foi de 30 unidades, o $\mathrm{R}^{2}$ da equação foi mais alto e atingiu a média de 16,23\%. Além disso, o coeficiente de regressão médio da característica foi semelhante em todas as situações analisadas, o que indica a tendência de que os grupos de contemporâneos nos quais existe valor muito alto de DIAS1, ou conversamente, valor muito baixo de GMD1, têm maior influência nos resultados. $\mathrm{O}$ fato de o $\mathrm{R}^{2}$ não ter sido tão alto significa que a variação da resposta foi muito grande nesses casos, mas que, em qualquer das situações, houve tendência de as contribuições do grupo de contemporâneos para os valores genéticos dos touros que dele participaram aumentarem conforme o valor máximo de DIAS1 daquele grupo.

No conjunto 1, com desvio-padrão simulado de 30 unidades, a média da contribuição de cada grupo para o valor genético dos animais foi de 11,77 unidades. O máximo de DIAS1 variou nos grupos de contemporâneos entre 2,0 e 10,0 unidades. Considerando esses extremos e o valor do coeficiente de regressão, a variação de $\mathrm{C}_{\mathrm{y}}$ atribuída ao valor máximo de DIAS1 ficou entre 0,36 e 1,80 e, assim, pode chegar a aproximadamente $12 \%$ da média de $C_{y}$, o que indica que o efeito do máximo pode ser importante, embora o $\mathrm{R}^{2}$ não tenha sido alto.

Na Figura 1 consta a distribuição dos valores de $C_{y}$ no máximo de DIAS1 em uma replicação tomada ao acaso do conjunto 3 com desvio-padrão 30.

Em algumas situações, os valores altos e baixos de $\mathrm{C}_{\mathrm{y}}$ ficaram separados por vazios nas regiões em que o máximo de DIAS1 foi mais alto. Em outras situações, isso não foi tão evidente, mas o mesmo fenômeno ocorreu em escala mais reduzida. Esses gráficos comprovaram que nestas regiões o máximo de DIAS1 foi uma observação discrepante e que 
Tabela 2 - Resumo das análises de regressão de $C_{y}$ no máximo, na média e no mínimo de DIAS1 em cada grupo de contemporâneos Table 2 - Summary of the regression analysis of $C_{y}$ on maximum, mean and minimum values of DIAS1 in each contemporary group

\begin{tabular}{|c|c|c|c|c|c|c|c|c|c|c|}
\hline \multirow[b]{2}{*}{ DPSIM $^{\mathrm{a}}$} & \multirow[b]{2}{*}{ Conj $^{b}$} & \multicolumn{3}{|c|}{$\begin{array}{l}\text { Máximo } \\
\text { Maximum }\end{array}$} & \multicolumn{3}{|c|}{$\begin{array}{l}\text { Média } \\
\text { Mean }\end{array}$} & \multicolumn{3}{|c|}{$\begin{array}{l}\text { Mínimo } \\
\text { Minimum }\end{array}$} \\
\hline & & $\mathrm{NR}^{\mathrm{c}}$ & $\mathrm{R}^{2 \mathrm{~d}}$ & $\mathrm{~b}^{\mathrm{e}}$ & $\mathrm{NR}^{\mathrm{c}}$ & $\mathrm{R}^{2 \mathrm{~d}}$ & $b^{e}$ & $\mathrm{NR}^{\mathrm{c}}$ & $\mathrm{R}^{2 \mathrm{~d}}$ & $\mathrm{~b}^{\mathrm{e}}$ \\
\hline 30 & I & 5 & 0,07 & 0,18 & 5 & 0,06 & 4,75 & 0 & 0,00 & $-0,09$ \\
\hline 20 & I & 5 & 0,03 & 0,16 & 5 & 0,01 & 2,31 & 2 & 0,00 & $-0,21$ \\
\hline 10 & I & 5 & 0,01 & 0,18 & 5 & 0,00 & 0,72 & 5 & 0,00 & $-0,22$ \\
\hline 30 & II & 5 & 0,11 & 0,17 & 5 & 0,08 & 2,54 & 2 & 0,00 & $-0,18$ \\
\hline 20 & II & 5 & 0,05 & 0,17 & 5 & 0,03 & 1,27 & 5 & 0,00 & $-0,18$ \\
\hline 10 & II & 5 & 0,02 & 0,19 & 5 & 0,00 & 0,46 & 5 & 0,00 & $-0,20$ \\
\hline 30 & III & 5 & 0,16 & 0,19 & 5 & 0,12 & 1,64 & 5 & 0,00 & $-0,21$ \\
\hline 20 & III & 5 & 0,08 & 0,19 & 5 & 0,04 & 0,84 & 5 & 0,00 & $-0,21$ \\
\hline 10 & III & 5 & 0,03 & 0,20 & 5 & 0,01 & 0,34 & 5 & 0,01 & $-0,20$ \\
\hline
\end{tabular}

${ }^{\mathrm{a}}$ Desvio-padrão simulado; ${ }^{\mathrm{b}}$ Conjuntos formados de acordo com a distribuição dos dados; ${ }^{\mathrm{c}}$ Número de regressões significativas; ${ }^{\mathrm{d}}$ Coeficiente de determinação;

e Coeficiente de regressão.

a Standard deviation simulation; ${ }^{b}$ Sets formed in agreement with the data distribution; ${ }^{c}$ Number of significant regressions; ${ }^{d}$ Coefficient of determination; ${ }^{e}$ Coefficient of regression

Tabela 3 - Resumo das análises de regressão de $C_{x}$ no máximo, na média e no mínimo de GMD1 em cada grupo de contemporâneos Table 3 - Summary of the regression analysis of $C_{x}$ on maximum, mean and minimum of GMD1 in each contemporary group

\begin{tabular}{|c|c|c|c|c|c|c|c|c|c|c|}
\hline \multirow[b]{2}{*}{ DPSIM $^{\mathrm{a}}$} & \multirow[b]{2}{*}{ Conj $^{b}$} & \multicolumn{3}{|c|}{$\begin{array}{l}\text { Máximo } \\
\text { Maximum }\end{array}$} & \multicolumn{3}{|c|}{$\begin{array}{l}\text { Média } \\
\text { Mean }\end{array}$} & \multicolumn{3}{|c|}{$\begin{array}{l}\text { Mínimo } \\
\text { Minimum }\end{array}$} \\
\hline & & $\mathrm{NR}^{\mathrm{c}}$ & $\mathrm{R}^{2 \mathrm{~d}}$ & $\mathrm{~b}^{\mathrm{e}}$ & $\mathrm{NR}^{\mathrm{c}}$ & $R^{2 d}$ & $\mathrm{~b}^{\mathrm{e}}$ & $\mathrm{NR}^{\mathrm{c}}$ & $\mathrm{R}^{2 \mathrm{~d}}$ & $b^{e}$ \\
\hline 20 & I & 5 & 0,00 & 0,17 & 0 & 0,00 & $-0,09$ & 5 & 0,00 & $-0,18$ \\
\hline 10 & I & 5 & 0,00 & 0,20 & 1 & 0,00 & 0,05 & 5 & 0,00 & $-0,18$ \\
\hline 30 & II & 5 & 0,01 & 0,20 & 0 & 0,00 & 0,01 & 5 & 0,01 & $-0,22$ \\
\hline 30 & III & 5 & 0,02 & 0,21 & 0 & 0,00 & 0,00 & 5 & 0,02 & $-0,22$ \\
\hline 20 & III & 5 & 0,02 & 0,21 & 0 & 0,00 & 0,01 & 5 & 0,02 & $-0,21$ \\
\hline 10 & III & 5 & 0,02 & 0,21 & 0 & 0,00 & 0,00 & 5 & 0,02 & $-0,21$ \\
\hline
\end{tabular}

${ }^{a}$ Desvio-padrão simulado; ${ }^{b}$ Conjuntos formados de acordo com a distribuição dos dados; ${ }^{c}$ Número de regressões significativas; ${ }^{d}$ Coeficiente de determinação e Coeficiente de regressão.

${ }^{a}$ Standard deviation simulation; ${ }^{b}$ Groups formed in agreement with the data distribution; ${ }^{c}$ Number of significant regressions; ${ }^{d}$ Coefficient of determination; ${ }^{e}$ Coefficient of regression.

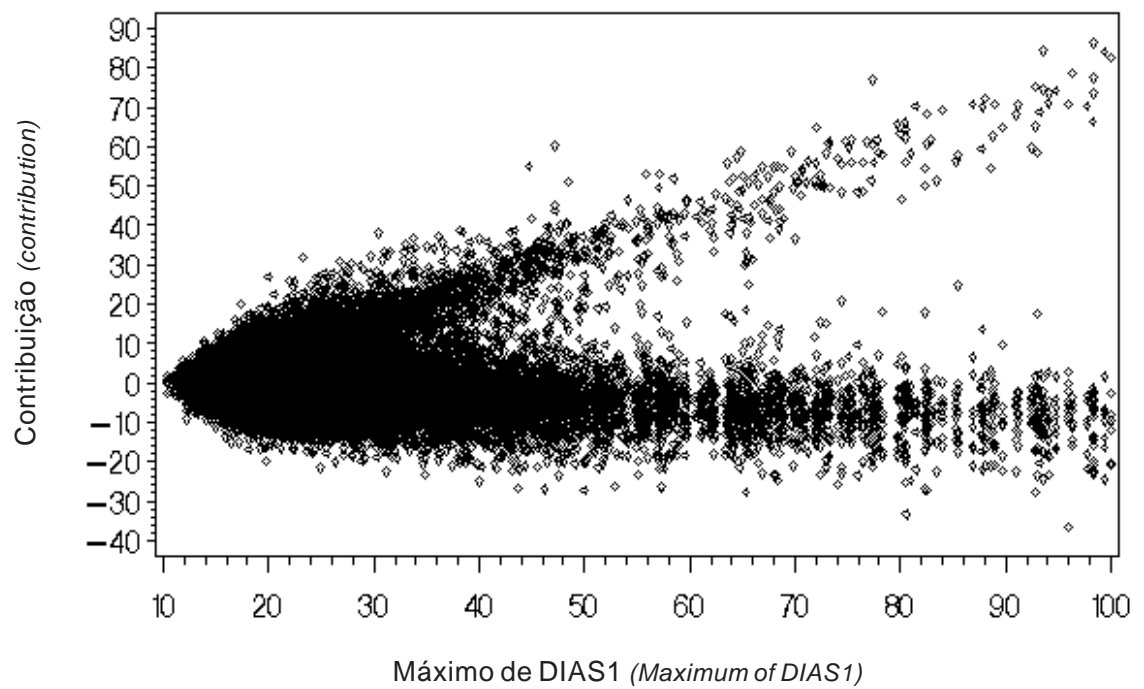

Figura 1 - Contribuição do grupo de contemporâneos para o valor do pai (C $\left.C_{y}\right)$ da inversa de uma variável com distribuição normal (DIAS1), de acordo com o valor máximo do grupo. Conjunto III - desvio-padrão 30.

Figure 1 - Contribution of the contemporary groups for the value of the sire $\left(C_{y}\right)$ of the inverse of a variable with normal distribution in agreement with the maximum value of the group. Set III - standard deviation 30 . 
a média do grupo para DIAS1 foi superestimada em decorrência dessa observação.

Como a progênie cujo touro está sendo avaliado não entrou na composição da média do grupo, o valor de $\mathrm{C}_{\mathrm{y}}$ daquele touro ficou muito desviado para cima e, dependendo da estrutura do grupo e do número de progênies do pai desse indivíduo, esse touro poderia ser determinante para o valor do pai na avaliação genética. Por outro lado, uma vez que para os outros pais a média ficou superestimada em virtude da presença deste valor muito alto, ocorrerá um desequilíbrio em indivíduos abaixo e acima da média nestes grupos. Finalmente, outra conseqüência seria a subestimação das médias dos filhos de outros animais e, em algumas situações, o desvio, que também pode ser determinante na formação do valor genético do pai. Pode ser observado na Figura 1 (conjunto 3, desvio-padrão 30) que, em grupos nos quais o valor do máximo de DIAS1 foi muito alto, não existiam indivíduos com valores intermediários de $\mathrm{C}_{\mathrm{y}}$.

No caso do melhoramento de bovinos, em grupos de contemporâneos em que existe um animal com valor de GMD muito baixo, o valor de DIAS desse animal será relativamente muito mais alto, o que afetará todos os animais do grupo, tanto em seu próprio valor, como, em menor escala, os pais de todos os animais presentes no grupo. $\mathrm{O}$ valor de DIAS será muito alto para o animal cujo filho tem valor discrepante e muito baixo para os outros animais no grupo. Assim, dependendo da estrutura do grupo e da variabilidade observada na característica, a maioria dos outros animais do grupo ficará, sejam superiores ou inferiores, com seus valores genéticos superestimados. Todo esse conjunto de efeitos depende de uma série de fatores e a mudança depende muito da estrutura do grupo, mas, de qualquer modo, em algumas situações essa mudança pode influenciar na classificação dos animais.

Quanto à média DIAS1 (Tabela 2), o efeito também foi significativo $(\mathrm{P}<0,01)$ para todas as replicações em todos os conjuntos analisados. Esse resultado era previsível se consideradas a dependência entre a média e a variância em DIAS1 e a dependência entre a média e o máximo. Contudo, o $\mathrm{R}^{2}$ nesses casos foi menor que na regressão sobre o máximo. O valor do coeficiente de regressão foi sempre maior que no caso anterior, mas os valores absolutos da média e sua variação foram bem menores que os observados no caso do máximo. Assim, parece que a média não foi tão importante quanto o máximo de DIAS1 na determinação da contribuição do grupo para o valor genético do indivíduo.

O valor mínimo de DIAS1, como previsto, teve pouca importância na determinação do valor de $\mathrm{C}_{\mathrm{y}}$ (Tabela 2). Considerando que a média aritmética de DIAS1 é proporcional à média harmônica de GMD1 (muito robusta quanto aos valores discrepantes à direita), valores muito altos de GMD1 não influenciariam significativamente o valor de $\mathrm{C}_{\mathrm{y}}$. As regressões foram significativas apenas em parte dos conjuntos 1 e 2 e em todo o conjunto 3. Nesse caso, quando os desvios-padrão foram menores, as regressões

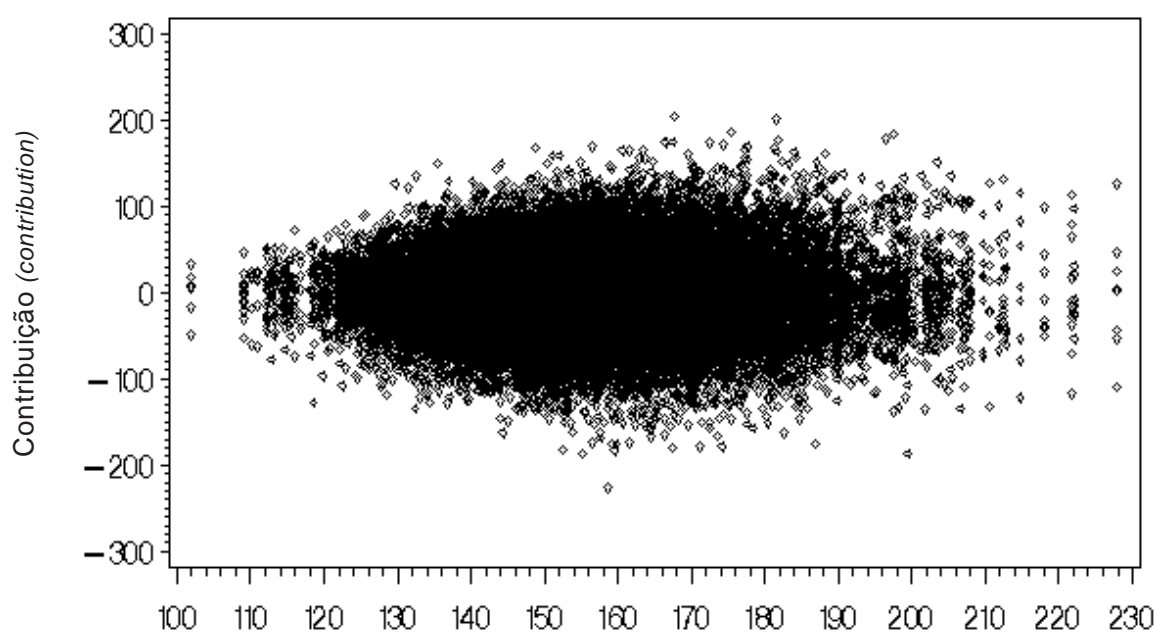

Máximo de de GMD1 (Maximum of GMD1)

Figura 2 - Contribuição do grupo de contemporâneos para o valor do pai (C $\mathrm{C}_{\mathrm{x}}$ ), de uma variável com distribuição normal (GMD1), de acordo com o valor máximo dessa variável no grupo. Conjunto III - desvio-padrão 30.

Figure 2 - Contribution of the contemporary groups for the value of the sire $\left(C_{x}\right)$, of a variable with normal distribution (GMD1) in agreement with the maximum value of this variable in the group. Set III - standard deviation 30. 
foram mais significativas e os $\mathrm{R}^{2}$ maiores (embora quase nulos).

Na Figura 2 consta a distribuição dos valores de $C_{x}$ no máximo de GMD1 em uma replicação tomada ao acaso em cada conjunto e desvio-padrão.

Os efeitos do máximo e do mínimo de GMD1 sobre $C_{\mathrm{x}}$ foram altamente significativos $(\mathrm{P}<0,01)$ em todas as replicações de todos os conjuntos. Apesar disso, os coeficientes de determinação foram muito próximos de zero em todas elas e o valor da regressão foi muito pequeno. O efeito do máximo de GMD1 sobre $\mathrm{C}_{\mathrm{x}}$ não apresentou tendência claramente definida (Figura 2). O efeito da média de GMD1 sobre $C_{x}$ não foi significativo na maioria das situações.

Esses resultados estão de acordo com o observado por Sirol et al. (2006), também em estudo de simulação. Esses autores verificaram que a distribuição da inversa de uma variável com distribuição normal era assimétrica e que sua variância era heterogênea, dependendo da média e do máximo observado na amostra. A assimetria da distribuição foi observada neste estudo e refletiu nas respostas também assimétricas, com efeito significativo do máximo e da média, mas não do mínimo de DIAS1 sobre o valor absoluto da contribuição, em contraste com o observado no caso do GMD1, em que as respostas foram simétricas. O efeito da heterogeneidade de variância sobre os resultados da avaliação genética são bem conhecidos (Garrick \& Van Vleck, 1987) e, no caso de dias para atingir determinado peso, os animais presentes em grupos nos quais a média ou o valor máximo da característica é muito alto, terão grande influência na avaliação genética de seus pais. Esse efeito foi mais pronunciado em situações em que o desvio-padrão era mais alto, visto que a ocorrência de valores mais baixos de GMD depende da variação da característica.

\section{Conclusões}

Em grupos de contemporâneos com um animal com valor de GMD muito baixo, o valor de DIAS desse animal será relativamente muito mais alto, o que afetará a média do grupo e os valores de todos os animais desse grupo. Esse efeito refletirá na avaliação de seus pais e será uma importante fonte de erros na avaliação genética do rebanho. Assim, a utilização de DIAS em substituição ao GMD como critério de seleção para o melhoramento de bovinos é contra-indicada, pois reduz a possibilidade de ganho genético para crescimento.

\section{Agradecimento}

À FAPESP, à Capes e ao CNPq, pelo financiamento do projeto e pela concessão das bolsas de pesquisa.

\section{Literatura Citada}

CARVALHEIRO, R.; MUNIZ, C.A.S.D.; QUEIROZ, S.A. et al. Estudo da simulação das relações entre a média aritmética, a média harmônica e o desvio-padrão. In: REUNIÃO ANUAL DA SOCIEDADE BRASILEIRA DE ZOOTECNIA, 38., 2001, Piracicaba. Anais... Piracicaba: Sociedade Brasileira de Zootecnia, 2001. p.626-627.

FRIES, L.A.; BRITO, F.V.; ALBUQUERQUE, L.G. Possíveis conseqüências de seleção para incrementar pesos às idadespadrão vs reduzir idades para produzir unidades de mercado. In: REUNIÃO ANUAL DA SOCIEDADE BRASILEIRA DE ZOOTECniA, 33., 1996, Fortaleza. Anais... Fortaleza: Sociedade Brasileira de Zootecnia, 1996. p.310-312.

GARRICK, D.J.; van VLECK, L.D. Aspects of selection for performance in several environments with heterogeneous variance. Journal of Animal Science, v.65, p.409-418, 1987.

MUNIZ, C.A.S.D.; CARVALHEIRO, R.; QUEIROZ, S.A. et al. Critérios de seleção baseado em médias aritméticas e médias harmônicas. In: REUNIÃO ANUAL DA SOCIEDADE BRASILEIRA DE ZOOTECNIA, 38., 2001, Piracicaba. Anais.. Piracicaba: Sociedade Brasileira de Zootecnia, 2001. p.629-631.

MUNIZ, C.A.S.D.; CARVALHEIRO, R.; FRIES, L.A. et al. Dois critérios de seleção na pré-desmama em bovinos da raça Gir. 2. Efeito na classificação dos animais. Revista Brasileira de Zootecnia, v.34, n.3, p.816-826, 2005.

ORTIZ PEÑA, C.D.; QUEIROZ, S.A.; FRIES, L.A. Análise comparativa de critérios de seleção para precocidade de crescimento pós-desmama em bovinos nelore no Paraguai. In: REUNIÃO ANUAL DA SOCIEDADE BRASILEIRA DE ZOotecniA, 37., 2000, Viçosa, MG. Anais... Viçosa, MG: Sociedade Brasileira de Zootecnia/Gnosis, [2000] 18par. (CD-ROM).

STATISTICAL ANALYSIS SYSTEM - SAS. User's guide: statistics. Versão 6.03. Cary: 1990. 584p.

SIROL, M.L.F.G.; HADLICH, J.C.; SUGUISAWA, L. et al. Efeitos da transformação de uma variável com distribuição normal em sua inversa sobre os parâmetros de sua distribuição usando técnicas de Monte Carlo. Revista Brasileira de Zootecnia v.35, n.3, p.1019-1029, 2006 (supl.).

Van VLECK, L.D.; BOLDMAN, K.G. Sequential transformation for multiple traits for estimation of (co)variance components with Derivative free algorithm for Restricted Maximum Likelihood. Journal of Animal Science, v.71, n.4, p.832 849, 1993. 